\title{
MAGNETIC FIELDS OF T TAURI STARS
}

\author{
EIKE W. GUENTHER \\ Thüringer Landessternwarte Tautenburg \\ D-07778 Tautenburg, Germany
}

\begin{abstract}
The magnetic field strengths of several T Tauri stars are derived by measuring the width of unblended $\mathrm{Fe} I$ lines of high and low values of $g_{\text {eff }} \cdot \lambda^{2}$ using the autocorrelation function. The T Tauri stars were selected for their low values of $v \cdot \sin i$, and large strengths of the Ca II emission component. The derived magnetic field strength are $2.0 \pm 0.6 \mathrm{kG}$ and $2.6 \pm 0.8 \mathrm{kG}$ for the classical T Tauri stars Lk Ca 15 and T Tau, respectively. An upper limit of $0.6 \pm 0.8 \mathrm{kG}$ is found for the weak-line $\mathrm{T}$ Tauri star Lk Ca 16. The method is tested by analysing two non-magnetic main sequence stars, and a late-type star that is known to have a strong magnetic field.
\end{abstract}

\section{Introduction}

Large observational and theoretical efforts have been invested in trying to understand how classical T Tauri stars (CTTS) can keep their low rotation rates while accreting matter. Currently, the most popular model is that strong magnetic fields $(\approx 1 k G)$ couple star and disk, so that angular momentum is transported outward, while matter is flowing inward (see reviews of F. Shu, and M. Camenzind in this volume).

Although strong magnetic fields might be highly important for CTTS, no field strength has been measured yet. However, there are some indications for the presence of magnetic fields in CTTS: The strongest evidence is the detection of circular polarized radio emission in HD 283447 (Phillips et al., 1996), and T Tauri (Phillips, 1993). However, both stars are binaries, and HD 283447 is intermittent between a weak-line T Tauri star (WTTS) and a CTTS. Although many more CTTS have been detected in the radio regime, the radiation is in general thermal (Bieging et al., 1984). As pointed out by André (1987), the non-detection of polarized radio emission 
in many CTTS does not necessarily exclude magnetic fields, because the ionized winds of CTTS are expected to free-free absorb any nonthermal radio emission produced near the surface of the star. Strong circular polarization of opposite helicity has also been detected in the two lobes of $\mathrm{T}$ Tau S (Ray et al., 1997). This is expected if the outflows are magnetically collimated. Additionally, there is some evidence that magnetic fields are important for the acceleration of the winds (Paatz \& Camenzind, 1996). Another argument for the presence of magnetic fields is that CTTS are relatively bright in X-rays, and that they occasionally show X-ray flares (Preibisch et al. 1993; Montmerle et al. 1983). Although some of the short time variability in the optical could be flares too, Gahm(1994) argues that most events are probably due to variations of the accretion rate and not due to flares. Another hint are the narrow emission line components (Ca II, He I) that originate most likely in plage regions on the surface of the star (Batalha et al., 1996). There are thus some indications that many CTTS have strong magnetic fields but this does not necessarily mean that the star and disk are magnetically coupled. Evidence for that comes from line profiles: (1) the observed large infall velocities can best be explained in the context of magnetic accretion (Edwards et al., 1994), and (2) the shape of the profiles can best be reproduced with models that assume that these lines are formed in magnetospheric infall zones (Hartmann et al., 1994).

The evidence for magnetic fields in WTTS is much stronger than for CTTS: Basri et al. (1992) detected a magnetic field of $1000 \pm 500$ gauss in the weak-line T Tauri star TAP35, and found an upper limit of $700 \mathrm{G}$ in Tap 10. Broad-band photometry implies that there are dark spots on WTTS. These spots cover areas of typically $5-40 \%$ of the stellar surface (Bertout 1989; Bouvier et al. 1993). In good agreement with this data are the results from Doppler imaging. Reconstructions of the surface markings clearly show cool spots on these stars (Joncour et al. 1994a, 1994b; Strassmeier et al. 1994; Rice \& Strassmeier 1996). More evidence comes from observations in the radio regime: At least some WTTS are non-thermal radio sources. The non-thermal nature of the radiation is evident from the circular polarization and from the large brightness temperature of $\geq 10^{7} \mathrm{~K}$. In general, the radio-emission of WTTS is consistent with gyrosynchroton radiation from a dipolar magnetic structure, and with a strength of the magnetic field of the order of one kG at the surface of the star (White et al., 1992). Additionally, VLBI-observations show that the sizes of the magnetospheres are of the order of $R_{\text {mag }} \approx 25 R_{*}$ in diameter (André $e l$ al., 1992). The correlation between the X-ray luminosity and the stellar rotation rate implies presence of a stellar dynamo (Bouvier, 1990), and flares have been observed in the radio X-ray and optical regime (White et al. 1992; Feigelson et al. 1985; Guenther \& Emerson 1997). Since CTTS 
are rather similar to WTTS, we may argue that CTTS should have strong magnetic fields, if WTTS have strong magnetic fields. However, Neuhäuser \& Preibisch (1994) argue that the larger X-ray fluxes (and faster rotation) of WTTS compared to CTTS implies a larger magnetic activity of WTTS. It can thus be concluded that there is some evidence for the presence of magnetic fields in CTTS and WTTS but it remains to be shown that CTTS and WTTS have magnetic fields with typical strength of kG. The aim of this work is to present measurements of the magnetic field strength in $\mathrm{T}$ Tauri stars.

\section{Methods to measure magnetic fields}

Since the magnetic field of a TTS will have its maximum magnetic flux density near the surface of the star and will decrease rapidly outwards, the strongest fields can be expected close to the star. Thus, magnetic field measurements should be carried out using photospheric lines, rather than emission lines. Since the photospheric spectrum of CTTSs is veiled, it will be easier to detect the fields in WTTSs. If the magnetic field structure is very complicated, observation of Stokes V will show no signal, because the signal from each magnetic north pole is canceled out by a magnetic south pole. However, the presence of many regions of opposite polarity will still lead to a broadening of the Stokes I-profile (Robinson et al., 1980), and to a change in the equivalent width of the lines.

\subsection{CHANGES OF THE EQUIVALENT WIDTH}

The presence of a magnetic field changes slightly the equivalent width of photospheric lines. Magnetic fields can thus be measured by measuring the equivalent width and modeling of the stellar atmosphere. The clear advantage of this method is that the equivalent width can be measured even in stars with relatively high values of $v \cdot \sin i$, and even when the lines are slightly blended, if only the whole spectrum is modeled. The disadvantage of this method is that a full radiative transfer code is needed and that the $g f$-values have to be precisely known. The $g f$-values are usually determined by measuring the equivalent widths in non-magnetic main sequence stars. The magnetic field is then derived by measuring the equivalent widths in the magnetic star by using the $g f$-values from the non-magnetic stars. Basri et al. (1992) used this method to measure a magnetic field of $1000 \pm 500$ gauss in the WTTS TAP35, and derived an upper limit for Tap 10 of $700 \mathrm{G}$. 


\subsection{THE LINE-BROADENING METHOD}

Another method is to measure the width of photospheric lines. Magnetic fields can simply be measured from the enhanced broadening of Zeeman sensitive lines over Zeeman insensitive lines. This is usually done by plotting $g_{\text {eff }} \cdot \lambda^{2}$ against the line width for lines from the flat part of the curve of growth (Preston 1971; Hensberge \& De Loore 1974). The slope of this curve then gives the magnetic field strength. The problem of the line-broadening method is that width of the lines has to be derived extremely accurately, and that the non-magnetic broadening of the lines could in principle be different for lines with different heights of formation. This would be the case, if the turbulence in the stellar atmosphere has a strong gradient. Although it can be expected that this effect is small, because the amplitude of the vertical component of the micro-turbulence decreases from just $2.5 \mathrm{~km} / \mathrm{s}$ to $1 \mathrm{~km} / \mathrm{s}$ when going from $\tau=1$ to $\tau=10^{-3}$ (Hollweger, 1967), and the macroturbulence is of the order of $2 \mathrm{~km} / \mathrm{s}$ only (Stix, 1989), it is probably safer to use lines originating in the same layers of the atmosphere (Robinson et al. 1980; Guenther \& Mattig 1991). This can be done by using lines of the same ion, of the same equivalent width, and in about the same spectral region. A disadvantage of this method is that it works better for slowly rotating stars. The most accurate way to measure the width of the lines is to calculate the autocorrelation functions for lines with large values of $g_{\text {eff }} \cdot \lambda^{2}$ and of lines with small values of $g_{\text {eff }} \cdot \lambda^{2}$ (Queloz et al., 1996). The magnetic field strength can then be derived from the difference of the width of the two autocorrelation functions. In the following, this method will be applied to unblended Fe I lines from the flat part of the curve of growth to derive magnetic field strengths of $\mathrm{T}$ Tauri stars.

\section{Selection criteria of the objects}

Since the aim of this work is detecting magnetic fields in WTTS and CTTS, it is better to analyse stars with a high probability for the presence of a strong magnetic field. For solar like stars, Schrijver et al. (1989) found a correlation between the flux of the emission core of the Ca II K line and the magnetic field strength. The flux of this component is related to the magnetic field strength, because the component is formed in plage regions. Since the emission core of the Ca II $8542 \AA$-line is formed in plage regions too, there should be a similar correlation for this line. Thus, stars with strong emission cores in the Ca II $8542 \AA$-line can be expected to have a strong magnetic field. The stars for this work where thus selected using the following criteria:

- small $v \cdot \sin i$ 


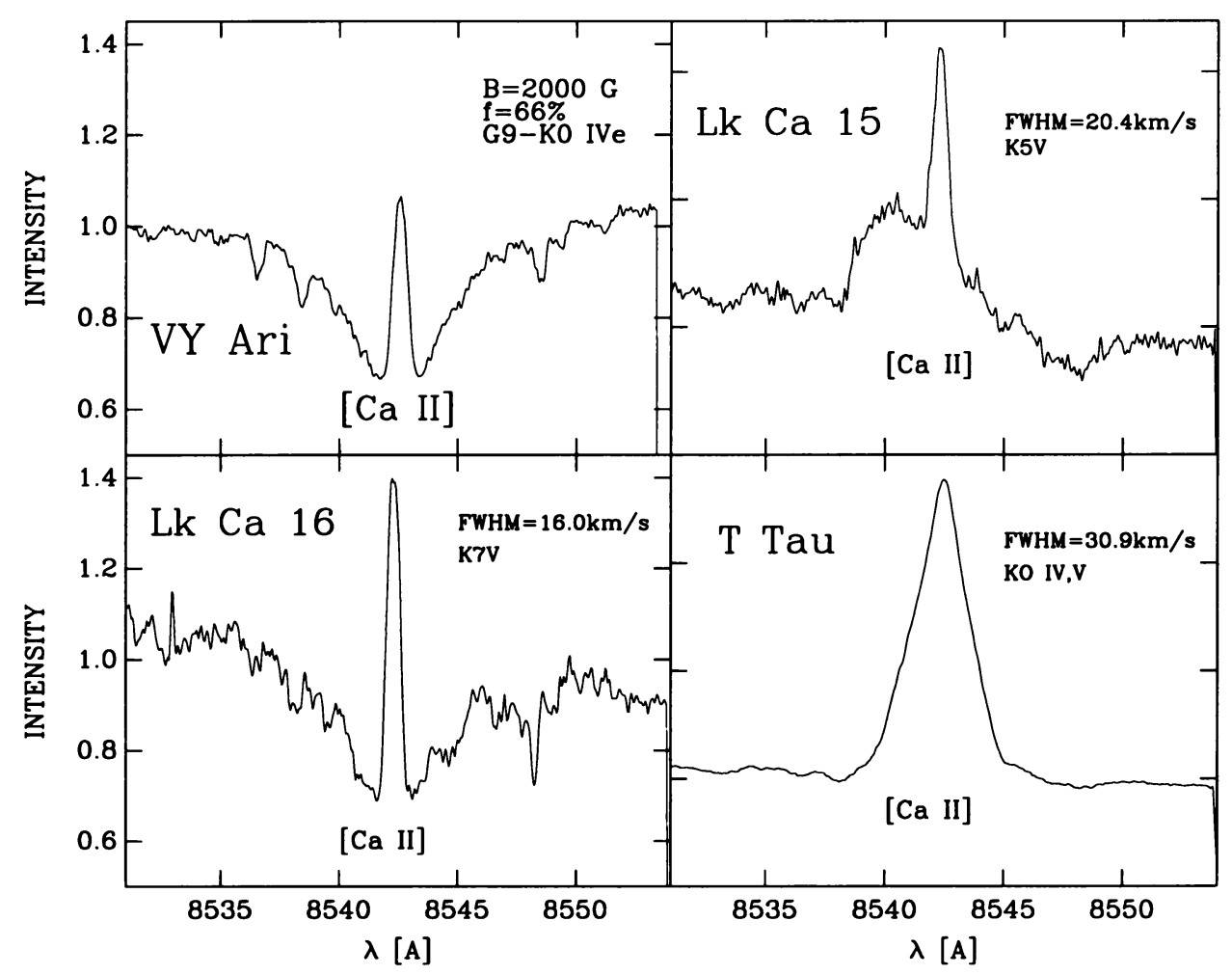

Figure 1. Ca II lines of the magnetic template star VY Ari, the WTTS Lk Ca 16, and the classical T Tauri stars Lk Ca 15 and T Tau. The narrow emission component seen in VY Ari originates in plage regions on the surface of the star, and is thus related to the strength of the magnetic field. This component is clearly visible in Lk Ca 15 and $\mathrm{Lk}$ Ca 16 too, making these stars suitable targets for the search of magnetic fields. Only a broad Ca II line is visible in $\mathrm{T}$ Tau.

- a large Ca II emission line component

- spectral type earlier than K7

- relatively large apparent brightness

- low veiling

Figure 1 shows the profiles of the Ca II $8542 \AA$-line of the sample. VY Ari is a star with a magnetic field strength of $2.8 \mathrm{kG}$ and a filling factor of $66 \%$ (Bopp et al., 1989). The emission line core is thus quite strong. It is interesting to note that the emission core of the Ca II lines is even stronger in the WTTS Lk Ca 16 than in VY Ari. Lk Ca 15 is intermittent between a CTTS and a WTTS. At the time of the observation we measured an equivalent width in $H \alpha$ of $24 \AA$, making it a CTTS at the time of the observations. The narrow component of the Ca II 8542 line is quite large. 


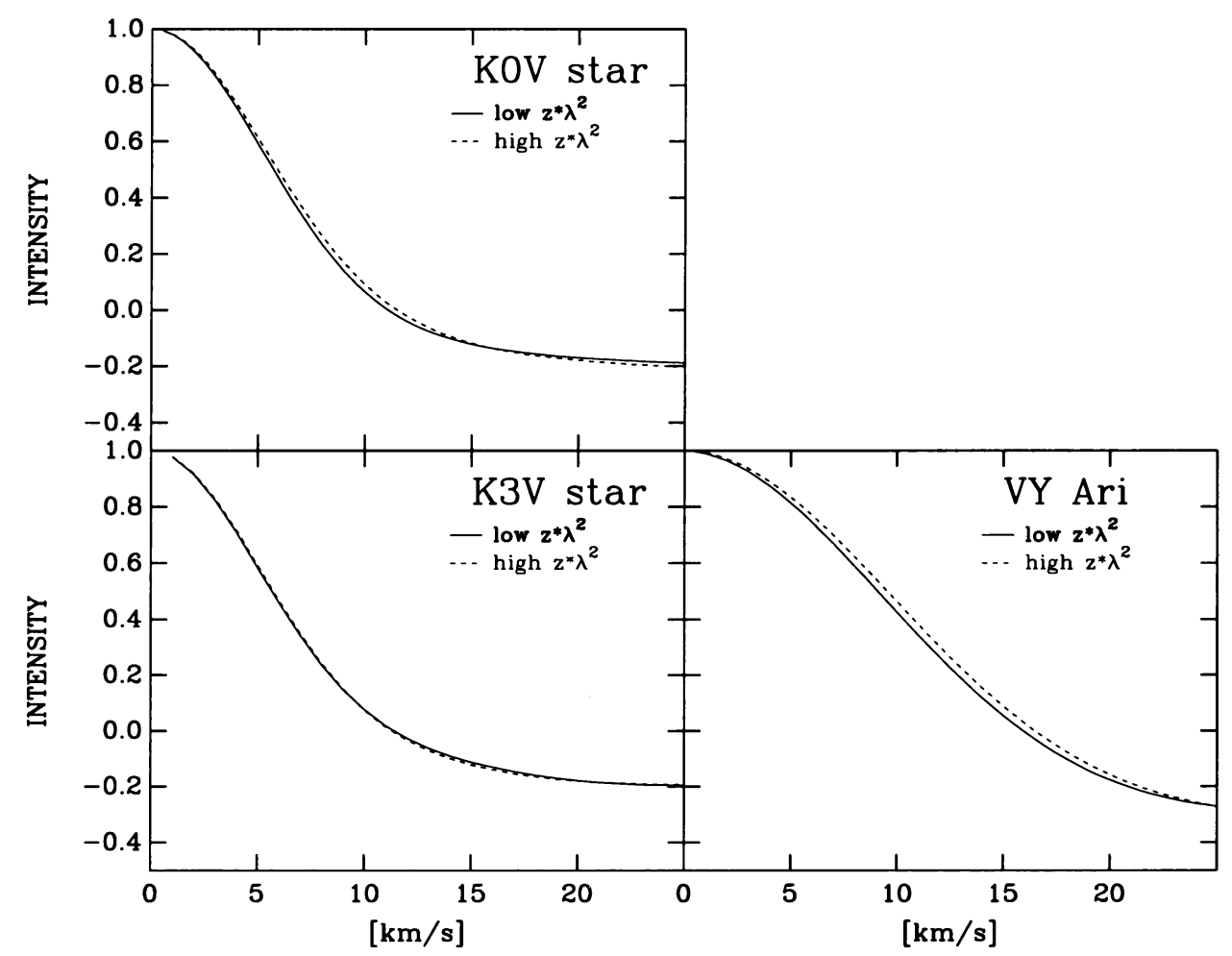

Figure 2. Autocorrelation functions of unblended FeI lines with low (full line) and high (dashed line) values of $g_{\text {eff }} \cdot \lambda^{2}$ of the main sequence stars HD 10780 (spectral type K0V), HD 16160 (spectral type K3V) and the magnetic template star VY Ari. The autocorrelation function of low and high $g_{e f f} \cdot \lambda^{2}$-values have exactly the same width in the case of HD 16160 indicating that no magnetic field could be detected in this star. In the case of HD 10780, the autocorrelation function for lines with high values of $g_{\text {eff }} \cdot \lambda^{2}$ is slightly broader than that of the lines with low values of $g_{e f f} \cdot \lambda^{2}$, indicating that there is a small magnetic field. In the case of VY Ari, the autocorrelation function for lines with high values of $g_{e f f} \cdot \lambda^{2}$ is significantly broader than that of the low $g_{e f f} \cdot \lambda^{2}$-values.

$\mathrm{T}$ Tauri was selected because of the relatively low veiling and the small $v \cdot \sin i$. The Ca II emission in T Tauri is just a broad emission line, and the narrow $\mathrm{Ca}$ II emission core is thus not visible.

\section{Measurements of the magnetic field strength}

Spectra of these stars were taken with the William Herschel Telescope using the University of Utrecht spectrograph. The resolution of the spectra is

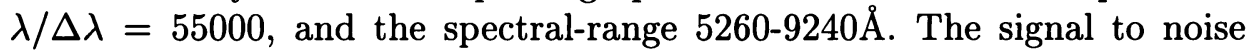
ratio is one-hundred or higher. The magnetic field strength is derived by measuring the width of the autocorrelation function of unblended Fe I lines 


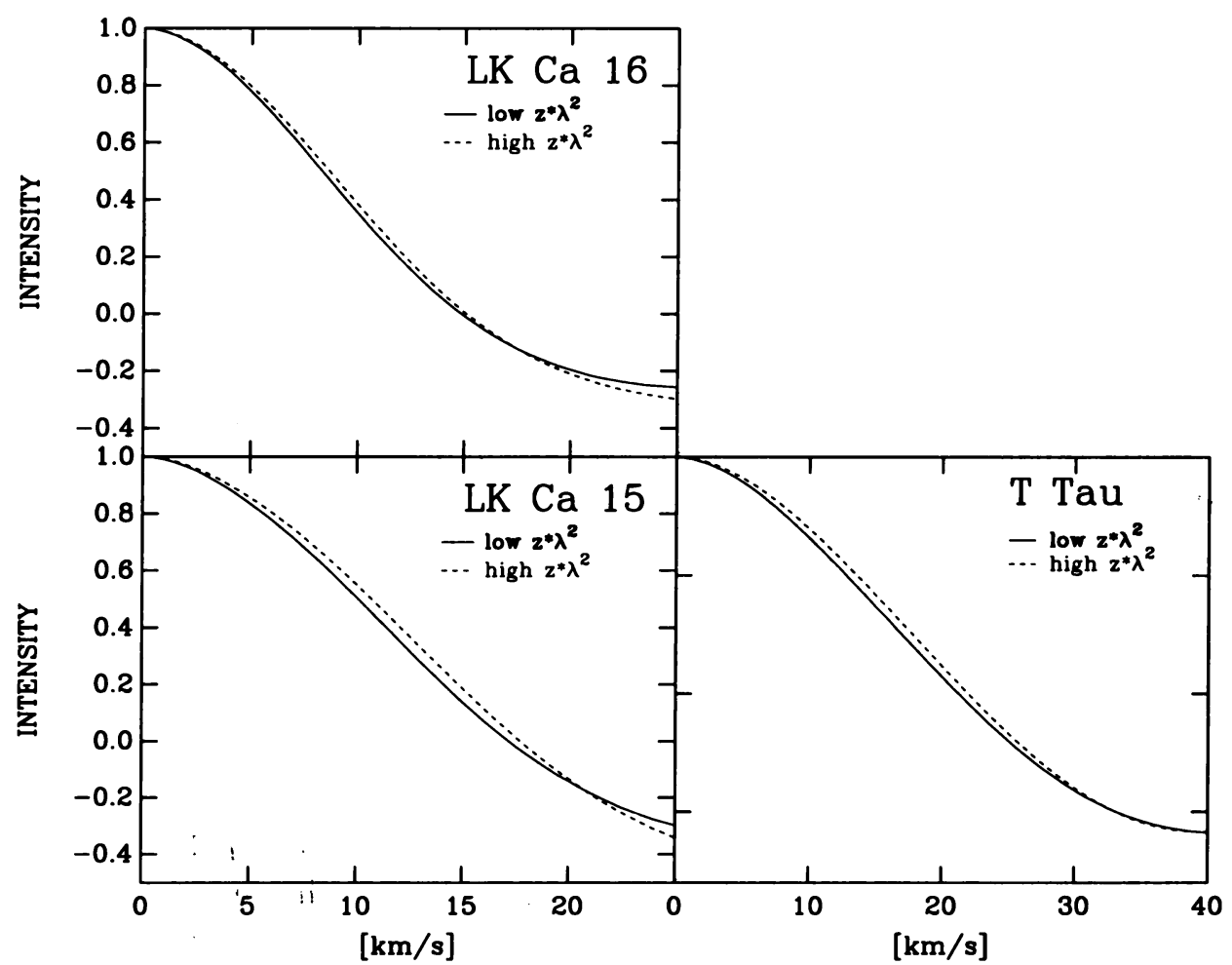

Figure 3. Like Figure 2 but for the WTTS Lk Ca 16, and the CTTS Lk Ca 15 and T Tauri. The autocorrelation function for lines with high value of $g_{e f f} \cdot \lambda^{2}$ is significantly broader than that of the the lines with low values of $g_{\text {eff }} \cdot \lambda^{2}$ in $\mathrm{Lk} 15$ and T Tau, indicating that a magnetic field has been detected in theses stars.

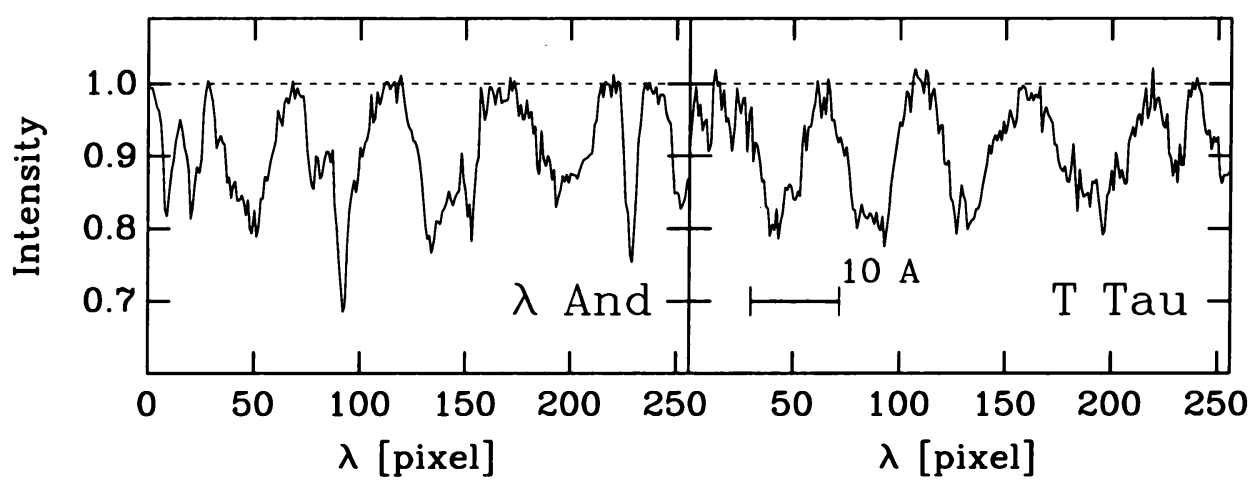

Figure 4. Spectrum of T Tau (right) and of the comparison star $\lambda$ And (left). Photospheric lines are clearly detected in $\mathrm{T}$ Tau. These lines could be used to measure the magnetic field strength in CTTS and WTTS. 
with a relatively high, and relatively low values of $g_{\text {eff }} \cdot \lambda^{2}$. A difference of $0.60 \mathrm{~km} / \mathrm{s}$ between the two autocorrelation functions corresponds to a magnetic field of one $\mathrm{kG}$. In order to test the method the width of the autocorrelation functions for the two main sequence stars HD 10780 and HD 16160 were measured. These stars are not expected to have strong magnetic fields. Additionally, the magnetic field of VY Ari was derived. Figure 2 shows the results. The autocorrelation function for low and high $g_{\text {eff }} \cdot \lambda^{2}$ values have exactly the same width in the case of HD 16160, indicating that no magnetic field could be detected in this star. In the case of HD 10780 , the autocorrelation function for lines with high value of $g_{e f f} \cdot \lambda^{2}$ is slightly broader than that of lines with low values of $g_{e f f} \cdot \lambda^{2}$. Thus, HD 10780 seems to have a small magnetic field of the order of $0.7 \pm 0.3 \mathrm{kG}$. In the case of VY Ari, the autocorrelation function for lines with a high value of $g_{e f f} \cdot \lambda^{2}$ is significantly broader than for lines with low $g_{e f f} \cdot \lambda^{2}$ values. The field strength of VY Ari is $1.3 \pm 0.6 \mathrm{kG}$. This corresponds well to the value of $\mathrm{B}=2000$ and $\mathrm{f}=66 \%$ from Bopp et al.(1989). Figure 3 shows the autocorrelation functions for the WTTS Lk Ca 16, and the CTTS Lk Ca 15 and T Tauri. The autocorrelation function for lines with high value of $g_{\text {eff }} \cdot \lambda^{2}$ is significantly broader than that for lines with low values of $g_{\text {eff }} \cdot \lambda^{2}$ in Lk Ca 15 and T Tau, indicating that a magnetic field has been detected in these stars. The corresponding field strength are $2.0 \pm 0.6 \mathrm{kG}$ and $2.6 \pm 0.8 \mathrm{kG}$, for Lk Ca 15 and T Tau, respectively. The errors of the values of the magnetic fields are derived from using different sets of lines and different algorithms in finding the continuum. No magnetic field could be detected in Lk Ca 16. Table 1 summarizes the results.

TABLE 1. Magnetic field strength

\begin{tabular}{lll}
\hline star & spec type & $f B[\mathrm{G}]$ \\
\hline HD 16160 & K3V & $\leq 0.2 \pm 0.2 \mathrm{kG}$ \\
HD 10780 & K0V & $0.7 \pm 0.3 \mathrm{kG}$ \\
VY Ari & G9-K0IVe & $1.3 \pm 0.6 \mathrm{kG}$ \\
Lk Ca 16 & K7V & $\leq 0.6 \pm 0.8 \mathrm{kG}$ \\
Lk Ca 15 & K5 & $2.0 \pm 0.6 \mathrm{kG}$ \\
T Tau & K0IV,V & $2.6 \pm 0.8 \mathrm{kG}$ \\
\hline
\end{tabular}

\section{Conclusions}

Magnetic fields with a strength of $2.0 \pm 0.6 \mathrm{kG}$ and $2.6 \pm 0.8 \mathrm{kG}$ have been detected for Lk Ca 15 and T Tau by measuring the width of unblended Fe I 
lines with different values of $g_{e f f} \cdot \lambda^{2}$. These are the first direct measurements of magnetic fields in classical T Tauri stars. It can thus be concluded that at least some classical T Tauri stars have strong magnetic fields. Although Lk Ca 16 has a strong Ca II emission line, no magnetic field has been detected.

\section{Outlook}

Since the magnetic broadening of the lines is $\propto g_{\text {eff }} \cdot \lambda^{2}$, it might be better to use lines in the infrared spectral region instead of the optical (although in practise, because of the increase of the intrinsic absorption line width with $\lambda$, the real gain is only $\propto \lambda$ (Giampapa et al., 1993)). Besides the gain in magnetic sensitivity, the larger brightness of these late type stars in the infrared will lead to relatively short exposure times, and the low extinction in the infrared might even allow to measure the magnetic field strength of some embedded sources.

The line broadening method would ideally be used on pairs of unblended lines originating from the same levels of the atmosphere, where one is a Landé factor $g_{\text {eff }}=0$ line, and the other is highly sensitive to the magnetic field. The only $g_{\text {eff }}=0$ lines in the near infrared region are the Fe I $1.00733 \mu \mathrm{m}$ (multiplet 1993) and the Fe I $1.01519 \mu \mathrm{m}$ (multiplet 1861) line (Nave et al., 1994). There are indeed two transitions from the same multiplets close by: the Fe I $1.00925 \mu \mathrm{m}$ (multiplet 1993), and the Fe I $1.01399 \mu \mathrm{m}$ (multiplet 1861) line. However, the Fe I $1.00925 \mu \mathrm{m}$ line has $g_{\text {eff }}=1.375$ $\left(7 \pi, 12 \sigma\right.$ components), and the Fe I $1.01399 \mu \mathrm{m}$ line has $g_{\text {eff }}=0.75(3 \pi, 6 \sigma$ components) only (Ramsauer et al. 1995; Nave et al. 1994).

The potential of the line pair Fe I $1.56572 \mu \mathrm{m}\left(g_{\text {eff }}=1.802\right)$ and Fe I $1.56528 \mu \mathrm{m}\left(g_{\text {eff }}=3.00\right)$ was pointed out by Solanki et al. (1992) in the context of the Sun. These two lines have very similar properties but differ in their sensitivity to the magnetic field. Guenther \& Emerson (1996) demonstrated that these lines are present in the spectra of WTTSs, and that the magnetic field strength can be determined with an accuracy better than $500 \mathrm{G}$ in relatively short integration times if $v$ sini $<20 \mathrm{~km} / \mathrm{s}$. Figure 4 shows a spectrum of the CTTS T Tauri and a comparison star in the same spectral region taken with the infrared camera MAGIC and the Coudé-spectrograph of the $2.2 \mathrm{~m}$ telescope on Calar Alto. The spectrum clearly shows that these photospheric lines are present in CTTS too. Thus, the potential of infrared lines for measuring magnetic fields in CTTS and WTTS is high, and infrared lines could be used for this kind of work in the future, especially if infrared echelle spectrographs become available.

\section{References}

André, P.: 1987, in Protostars and Molecular Clouds, eds. Montmerle \& C. Bertout 
(Saclay: CEA-Doc), 143

André, P., Deeney, B.D., Phillips, R.B., Lestrade, J.-F.: 1992, ApJ 401, 667

Basri, G., Marcy, G. W., Valenti, J.A.: 1992, ApJ 390, 622

Batalha, C.C, Stout-Batalha, N.M., Basri, G., Terra, M.A.O.: 1996, ApJ Suppl. 103, 211

Bertout, C.: 1989, ARA\&A 27, 351

Bieging, J.H., Cohen, M., Schwartz, P.R.: 1984, ApJ 282, 699

Bopp, B.W., Saar, S.H., Ambruster, C., Feldman, P., Dempsey, R., Allen, M., Barden, S.P.: 1989, ApJ 339, 1059

Bouvier, J.: 1990, AJ 99, 946

Bouvier, J., Cabrit, Fernandez, M., Martin, E.L., Matthews, J.M.: 1993, A\&A 272, 176

Edwards, S., Hartigan, P., Ghandour, L., Andrulis, C.: 1994, AJ 108, 1056

Feigelson, E.D., Montmerle, T.: 1985, ApJ 289, L19

Gahm G.F: 1994, in Flares and Flashes, Proceedings of IAU Colloqium No.151, J. Greiner,

H. Duerbeck, R.E. Gershberg (eds.), Springer Heidelberg, p. 203

Giampapa, M.S., Colub, L., Worden, S.P.: 1983, ApJ 268, L121

Guenther, E. Mattig, W.: 1991, A\&A 243, 244

Guenther, E.W., Emerson, J.P.: 1996, A\&A 309, 777

Guenther, E.W., Emerson, J.P.: 1997, A\&A in press

Hartmann, L., Hewett, R., Calvet, N.: 1994, ApJ 426, 669

Hatzes, A.P.: 1995, ApJ 451, 784

Hensberge, H., De Loore, C.: 1974, A\&A 37, 367

Hollweger, H.: 1967, Z. Astrophys. 22, 265

Joncour, I., Bertout, C., Bouvier, C.: 1994a, A\&A 291, L19

Joncour, I., Bertout, C., Bouvier, C.: 1994b, A\&A 285, L25

Montmerle, T., Koch-Miramond, L., Flaarone, E., Grindlay, J.E.: 1983, ApJ 269, 182

Nave G., Johansson, S., Learner, R.C.M., Thorne, A.P.: 1994, ApJS 94, 221

Neuhäuser, R., Preibisch, T.: 1994, in Flares and Flashes, Proceedings of IAU Colloqium No.151, J. Greiner, H. Duerbeck, R.E. Gershberg (eds.), Spinger Heidelberg, p. 216

Paatz G., Camenzind M.: 1996 A\&A 308, 77

Phillips, R., Lonsdale, C.J., Feigelson, E.D.: 1993, ApJ 403, L43

Phillips, Lonsdale, C.J., Feigelson, E.D., Deeney, B.D.: 1996 AJ 111, 918

Preibisch, Th., Zinnecker, H., Schmitt, J.H.M.M.: 1993, A\&A 279, L33

Preston, G.W.: 1971, ApJ 164, 309

Queloz, D., Babel, J., Mayor, M.: 1996, in Cool Stars, Stellar Systems, and the Sun 9th Cambridge Workshop, ASP Conference Series Vol 109, R. Pallavicini, A.K. Dupree (eds.), p. 627

Ramsauer, J., Solanki, S.K., Biémont, E.: 1995, A\&AS, 113, 71

Ray T.P., Muxlow, T.W.B., Axon, D.J., Brown, A., Corcoran, D., Dyson, J., Mundt, R.: 1997, Nature 385, 415

Rice, J.B, Strassmeier, K.G.: 1996, A\&A 316, 164

Robinson, R.D., Worden, S.P., Harvey, J.W.: 1980, ApJ 236, L155

Schrijver, C.J., Coté, J., Zwaan, C., Saar, S.H.: 1989, ApJ 337, 964

Solanki, S.K., Rüdi, I., Livingston, W.: 1992, A\&A 263, 312

Stix, M.: 1989 The Sun, Springer Heidelberg, p.142

Strassmeier, K.G., Welty, A.D., Rice, J.B.: 1994, A\&A 285, L17

White, S.M., Pallavicini, R., Kundu, M.R.: 1992, A\&A 259, 149 